\title{
La incidencia del PROGRESAR en la trayectoria académica de jóvenes argentinos. Un estudio de caso
}

The effects of the PROGRESAR program in Argentine's young students. A case study

Maria Esther Lara y Luisa María Salazar Acosta

Universidad Nacional de Salta (Argentina)

lara.mariaesther@gmail.com, salazarluisamaria@hotmail.com

\section{Resumen}

Este artículo presenta resultados de una investigación acerca de la incidencia del PROGRESAR en las trayectorias académicas-formativas de los jóvenes estudiantes de una institución de nivel superior no universitario del departamento de Metán, provincia de Salta, Argentina, durante 2015. Dicha investigación permite recuperar las percepciones de las usuarias y los usuarios del programa, respecto a la incidencia en el nivel académico-formativo, institucional y familiar. El estudio se basa en un enfoque interpretativo, apoyado en un estudio de casos. Se utilizó la entrevista semi-estructurada para la recolección de información y se privilegió el análisis de datos cualitativos. Los resultados evidencian que el programa posee relevancia para aquellos jóvenes que no disponían de recursos económicos para acceder a 
estudios superiores. Se evidencia también la incidencia a nivel familiar, dado que permitió aliviar los gastos en los hogares. Además, existe incidencia institucional en cuanto al aumento de matrícula y las dificultades de albergar a una población, sin políticas edilicias que respalden a las instituciones formadoras. En cuanto a la trayectoria académica, existe incidencia para aquellos que lo utilizan para fines educativos. Cabe señalar que no existe un control y seguimiento riguroso de los usuarios, lo cual es considerado como una debilidad del programa.

Palabras claves: PROGRESAR; trayectorias académicas; jóvenes; estudios de casos.

\section{Abstract}

This article presents results of a qualitative research about the incidence of the PROGRESAR program in the academic-formative trajectories of the young students of a Non-University Higher Education Institution of the department Metán, province of Salta, Argentina during 2015. This research Allows to recover the perceptions of the institutional actors - and of the users of the program in particular -, regarding the incidence of the same in academic-formative, institutional and familiar level. The study is based on an interpretative approach, based on a case study. Semi-structured interviews were used for data collection and qualitative data analysis was emphasized. The results show that the program has relevance for those young people who did not have the economic resources to access higher education. It is also evident the incidence at the family level, since it allowed to alleviate the familiar expenses in homes. In addition, there is an institutional impact on the increase in enrollment and the difficulties of housing a population without building policies that support the training institutions. As for the academic trajectory, there is an incidence for those who use the economic benefit for educational purposes. Note that no there is a control and monitoring of users, which is considered a weakness of the program.

Keywords: PROGRESAR; academic career; young; case studies.

La presente investigación indaga sobre la incidencia del Programa de Respaldo a Estudiantes Argentinos (PROGRESAR) en el trayecto académico-formativo, personal y familiar, de estudiantes del Instituto de Educación Superior Nro. 6021 de la localidad de San José de Metán, departamento de Metán, provincia de Salta, en 2015. Para ello, las unidades de análisis fueron estudiantes usuarios del PROGRESAR que cursan alguna carrera (al momento del 
estudio) en dicha institución. Se busca conocer cuál es la opinión que tienen estos jóvenes sobre el programa y de qué manera incidió en su vida personal, académica e institucional.

Metán, es una ciudad situada al sureste de la provincia de Salta. Se encuentra a 170 kilómetros de la ciudad capital. Según el Censo 2010, Metán cuenta con 40.351 habitantes. Concentra actividades gubernamentales, tribunales, cárcel, Unidad Regional de la Policía y otras dependencias estatales, como ser escuelas primarias, secundarias, educación inicial, una escuela de Educación Especial, dos Institutos de Educación Superior no Universitario (uno de gestión privada y otro de gestión estatal), y dos sedes universitarias.

Los objetivos del estudio consistieron en indagar, a través de las voces de las y los estudiantes, la incidencia del PROGRESAR en el instituto de referencia y en los sujetos. Además, se planteó el propósito de conocer percepciones y representaciones de los diferentes actores institucionales y particularmente de las y los estudiantes que perciben el PROGRESAR en el Instituto de Educación Superior Nro. 6021. Y por último, analizar las generalidades y particularidades del PROGRESAR en el IES Nro. 6021 "Juan Carlos Dávalos" y en los sujetos.

En la investigación se trabajó desde un enfoque de investigación cualitativa, de corte interpretativo, basado en un estudio de casos. Se buscó conocer cuestiones generales y particulares sobre las y los estudiantes del IES Nro. 6021, que son usuarias y usuarios del programa. La interpretación de esos datos permitió dar cuenta, desde la perspectiva de los actores, de cuál fue la incidencia de dicho programa en la vida de los mismos.

EI PROGRESAR constituye una política social relativamente reciente. Mucho se habla en los medios acerca de los programas en general y del PROGRESAR en particular. Sin embargo, existen pocas investigaciones acerca del impacto en todo el país, sobre todo en el nivel de educación superior universitario y no universitario. Este informe se focaliza, entonces, en la incidencia del PROGRESAR en los institutos de educación superior no universitarios y se propone además, generar conocimiento sobre el PROGRESAR y contribuir a futuras investigaciones respecto de la temática.

\section{EI PROGRESAR, los jóvenes y sus trayectorias}

EI PROGRESAR forma parte del Programa de Inclusión laboral y productiva dentro de los Programas de Transferencias Condicionadas. Surge el 23 de enero de 2014 por Decreto nacional Nro. 84/2014, el cual tiene como antecedentes diversos programas y políticas sociales que impulsó el gobierno nacional de turno para cumplimentar con el lema de la inclusión social y educativa en las y los jóvenes argentinos. Dicho programa apunta a generar oportunidades educativas y laborales a las y los jóvenes entre 18 y 24 años de edad, con el objetivo de iniciar y/o continuar los estudios superiores (PEN, 2014). 
En sus inicios, el PROGRESAR consistía en una prestación económica universal de seiscientos pesos mensuales ( $\$ 600)$, no contributiva, destinada a brindar apoyo económico a jóvenes desocupadas y desocupados o trabajadores y trabajadoras informales, cuyo ingreso sea inferior o igual al salario mínimo, vital y móvil (\$4.400). El 12 de marzo de 2015 se anuncian cambios en el programa: la prestación económica pasa de $\$ 600$ a $\$ 900$ mensuales; además aumenta el ingreso tope del titular y su familia, el cual varía de $\$ 4.716$ a $\$ 14.148$.

El monto de la prestación es de \$900. Se paga el 80\% (\$720) a partir de la inscripción y se retiene un 20\% (\$180) todos los meses. En los meses de marzo, julio y noviembre de cada año, las y los usuarios deben presentar un certificado que acredite que siguen estudiando. Cada vez que presenten un certificado, se les paga el $20 \%$ retenido en el cuatrimestre.

El decreto del PROGRESAR establece acciones orientadas a apoyar a las usuarias y los usuarios en la continuación de sus estudios, vinculación con actividades de capacitación y programas de inserción laboral y provisión de lugares para el cuidado de los hijos e hijas de los usuarios que estén en edad pre-escolar, para facilitar su asistencia a las sesiones de capacitación laboral. Por último, ofrece una red de tutorías para apoyar y orientar el proceso formativo de los/as usuarios/as.

La prestación es financiada con recursos del Tesoro Nacional, para lo cual la Jefatura de gabinete de Ministros deberá prever las adecuaciones presupuestarias pertinentes para el ejercicio en curso. La administración, gestión, otorgamiento y pago de las prestaciones que resulten de la aplicación del PROGRESAR estarán a cargo de la Administración Nacional de la Seguridad Social (ANSES), quedando facultada para dictar las normas interpretativas, complementarias y aclaratorias necesarias para su implementación.

Dado que la mirada del análisis está puesto en una política juvenil, como el caso del programa PROGRESAR, es necesario plantear algunos parámetros que sirven de ejes conceptuales sobre los que apoyar la lectura interpretativa del presente artículo. En relación con la temática que interesa, es fundamental considerar el concepto de juventud que, como categoría compleja, amerita una sección aparte, aún más si nos referimos a las políticas públicas focalizadas respecto de la juventud durante este contexto histórico.

La conceptualización de juventud como sujeto social presenta un desafío en el momento de delimitar las fronteras que permitan caracterizarla como categoría. La edad y el sexo son bases de clasificaciones sociales y estructuraciones de sentido; ellas aparecen en todas las sociedades como uno de los ejes ordenadores de la actividad social. En este sentido, Bourdieu señala que "las divisiones entre las edades son arbitrarias" (Gutiérrez, 2000: 163).

Siguiendo la misma línea, Margulis y Urresti (1996) expresan que resulta imprescindible contextualizar el concepto juventud con la multiplicidad de situaciones en que esta etapa de la vida se desarrolla, situándolo en un contexto socio-histórico en el que se desenvuelve y condiciona la manera de ser joven. Las diferentes concepciones en torno a la juventud van 
modificándose conforme a la situación histórica y el papel que desempeñan los y las jóvenes en una determinada sociedad y en función a la matriz cultural predominante. Al hablar de juventud, la literatura sociológica incorpora en los análisis la diferenciación social y hasta cierto punto la cultura. Entonces, se dice que la "juventud depende de una moratoria, un espacio de posibilidades abierto a ciertos sectores sociales y determinados a determinados periodos históricos" (Margulis y Urresti, 1996: 2). Se evidencia que la condición socio-histórico cultural no se ofrece de igual forma para todos los que componen la categoría estadística joven. Es por ello que hablamos de juventudes, dado que las condiciones no son las mismas para todos los sujetos por igual.

La consolidación de la juventud como sujeto social, con caracteres heterogéneos y potencialidades que le son propias y, lejos de los enfoques limitados a la definición como mero recorte etario, ha atravesado un largo y complejo recorrido (Rodríguez Use y Giambroni, 2015). Actualmente, el sector joven ha sido reconocido y es destinatario de políticas públicas frente a la realidad social, económica y política en el que se desenvuelven.

En los últimos años se ha buscado abordar la problemática desde un enfoque multidimensional. Asimismo, esta diversidad de aproximaciones hacia los jóvenes hace imposible definir un modelo ideal de programas o políticas de juventud que garanticen un correcto abordaje de sus problemáticas. Las políticas de juventud del siglo XXI, por tanto, deben tener en cuenta no sólo los cambios en cuanto a la cultura joven y sus trayectorias reales hacia la adultez, sino también los problemas de la economía y su incidencia en ellos.

Por otro lado, las trayectorias de los estudiantes son entendidas como expresión de la articulación entre el pasado incorporado por los actores y las experiencias de socialización vividas, las condiciones socioculturales, las propuestas y oportunidades educativas y laborales disponibles en su propio contexto y las elecciones propias. De igual modo, se tratan como procesos insertos en un marco histórico social de transformaciones y cambios en la condición juvenil, situación que opera condicionando sus recorridos y sus prácticas. Las trayectorias son analizadas también como procesos abiertos, en tanto proyecto. En ellas, el tiempo presente no está determinado solamente por las experiencias acumuladas del pasado del sujeto, sino que también forman parte de él las aspiraciones y los planes para el futuro: el presente aparece condicionado por los proyectos o la anticipación del futuro (Dávila León, 2004).

La formación, en tanto trayectoria, centra su mirada en el sujeto en formación y compromete la intervención de las instituciones formadoras donde se lleva a cabo. El trayecto formativo de los estudiantes de educación superior comienza con su ingreso en una institución. Sin embargo, como señala Cols (2008), las trayectorias de formación "se engarzan con otras trayectorias". En efecto, "en el marco de las Instituciones de Educación Superior los y las estudiantes llevan a cabo un itinerario en tanto tales, pero este recorrido se cruza con otras trayectorias anteriores y paralelas, ligadas a su vida escolar, familiar, laboral, política y cultural” (Cols, 2008: 6). 


\section{Consideraciones metodológicas}

El presente informe está enmarcado en una tesis de grado, en la que se trabajó desde un enfoque de investigación cualitativa, basada en un estudio de casos. Se buscó conocer cuestiones generales y particulares sobre las y los estudiantes del Instituto de Educación Superior Nro. 6021, que son usuarias y usuarios del PROGRESAR. La interpretación de esos datos permitió dar cuenta, desde la perspectiva de las y los jóvenes, cuál fue la incidencia de dicho programa en la vida de los mismos.

El estudio se elaboró con el propósito de conocer la incidencia del programa en el Instituto de Educación Superior Nro. 6021, particularmente e indagar acerca de las percepciones de las usuarias y los usuarios del programa. Para ello se trabajó con una selección de casos, con el fin de obtener una descripción sobre la incidencia del PROGRESAR en el Instituto.

Para el presente estudio se seleccionó la mencionada institución, a fin de profundizar en el análisis sobre la incidencia de un programa nacional, dado que es el único Instituto de Educación Superior no Universitario en la localidad, de gestión estatal y público. Para ello se realizaron entrevistas semi-estructuradas a los actores institucionales, para recoger sus propias voces, percepciones y opiniones acerca del PROGRESAR y su incidencia en la vida personal, familiar y académica. Esto permitió describir e interpretar el objeto de investigación, buscando una comprensión a partir de un estudio de casos. Con este propósito, la selección de las entrevistadas y los entrevistados se basó en el potencial informativo de cada "caso", ya que se trata de desarrollar comprensiones sobre un fenómeno particular.

Los informantes fueron siete estudiantes usuarias y usuarios del PROGRESAR, que cursaban algunas carreras de Formación Docente, dado que interesaba indagar acerca de la incidencia de dicho programa en su trayecto académico-formativo, personal y familiar. También se realizaron entrevistas a la Vicerrectora para indagar acerca de la incidencia que tiene el PROGRESAR en el Instituto de formación docente, en relación a la matrícula, el espacio físico y el trayecto académico de las y los estudiantes usuarios.

Se realizó una lectura y relectura de las transcripciones de las entrevistas y se inició el proceso de estructuración y organización de las mismas dentro de las respectivas categorías, lo cual permitió la comprensión del problema de investigación y focalización de la temática planteada. Seguidamente, se elaboraron categorías de análisis que son "sin dudas una importante herramienta en el análisis de datos cualitativos (Rodríguez Gómez, Gil Flores y García Jiménez, 1996: 208). Luego, se realizó un segundo nivel de análisis, respondiendo a una codificación más compleja. En esta instancia se revisó el marco teórico de referencia y se elaboraron, a partir del análisis, las propias teorías. 


\section{Importancia del programa en la vida de las y los usuarios y opinión de la familia respecto al PROGRESAR}

EI PROGRESAR surge con la finalidad de incluir educativamente y socialmente a los jóvenes argentinos de 18 a 24 años. El mismo se implementa en 2014 y, pese a que es una política educativa reciente, puede evidenciarse la incidencia del programa en las y los jóvenes usuarios. La importancia del programa en la vida de los sujetos se evidencia en la posibilidad de acceder a los estudios superiores y además poder estudiar en la localidad, sin necesidad de emigrar a la capital salteña o a provincias vecinas. Manifiestan la diversidad de ofertas educativas que posee el Instituto y otras instituciones educativas del medio, lo cual facilita y promueve el acceso a los niveles superiores.

La plena titularidad de derechos económicos y sociales requiere de programas que promuevan la integración e inclusión sociales. Estos deben permitir la formación de capital humano y social, y actuar sobre el entorno familiar y comunitario recurriendo a diversos mecanismos. Importa, en el caso de las jóvenes madres solteras, que tienen la posibilidad de estudiar en su ciudad natal y cuidar a sus hijos allí.

Se evidencia la situación de las jóvenes y los jóvenes usuarios con hijos a cargo, y la situación de las y los jóvenes sin hijos, que poseen una moratoria social y vital (Margulis y Urresti, 1994) para dedicarse exclusivamente a los estudios. Los testimonios dan cuenta de múltiples realidades del ser joven, lo cual permite efectivizar el PROGRESAR en manos de los jóvenes en condiciones más vulnerables.

La juventud no es independiente del género (Ibídem). La juventud tiene otras implicancias para el género femenino. Esto implica una diferenciación más en cuanto a la condición de ser joven y mujer. Pueden evidenciarse las características que adoptan las jóvenes madres y el apoyo que reciben de la familia para salir adelante. Las políticas de juventud, frente a situaciones de vulnerabilidad, deben generar mecanismos de inclusión social, laboral y educativa.

En relación con ello, la opinión de la familia respecto a la política del PROGRESAR fue tangible. Todos coinciden en que el programa permite solventar los estudios superiores y otros gastos, permitiendo al usuario ingresar, continuar y culminar los estudios en una institución de nivel superior no universitario. En este sentido, la familia fue el sostén y el gestor para informarse y solicitar el derecho para el bienestar académico y personal de los jóvenes. Por otro lado, la importancia del programa según las opiniones de la familia fue la de alivianar los gastos de mantenimiento en la formación del estudiante, haciendo hincapié en la oportunidad que tienen de estudiar. La familia considera oportuna la prestación económica del PROGRESAR. 
EI PROGRESAR adquiere importancia, entonces, gracias al estímulo y la voluntad de las y los jóvenes usuarios, en particular, y la familia en general. La importancia del programa es contundente en la opinión de la familia. El monto económico permitió alivianar gastos destinados tradicionalmente a la formación académica de los jóvenes, además de ser una ayuda para las necesidades básicas de la familia. Esto permite demostrar que la incidencia del PROGRESAR no sólo ha sido positiva para los jóvenes usuarios, sino también para sus familias.

La importancia del programa en la vida de las y los jóvenes usuarios y en su entorno familiar ha sido relevante. EI PROGRESAR apunta a la "necesidad de un acceso real y flexible a la oferta educativa de los jóvenes" (PEN, 2014). Las condiciones de oferta educativa permiten la inclusión educativa de las y los jóvenes que optan por cursar estudios superiores en la localidad de Metán.

\section{Las fortalezas del PROGRESAR según los actores y la inclusión educativa como finalidad del programa}

Desde 2009 el Estado nacional adoptó políticas públicas que tenían como finalidad mejorar la situación de los grupos familiares en situación de vulnerabilidad social, fomentando de esta manera la inclusión social y educativa. EI PROGRESAR, como política juvenil, busca atender a las particularidades de sus problemáticas, garantizando los derechos sociales y permitiendo el ejercicio pleno de la ciudadanía.

La noción de inclusión significa, como punto de partida, el reconocimiento de una sociedad diversa y heterogénea y que la misma debe ser revalorizada. En el ámbito educativo, la inclusión educativa supone el derecho al aprendizaje por parte de todas y todos, independientemente de sus características individuales, con el fin de proporcionar atención al conjunto de demandantes, según sus propias necesidades, lo que implica "velar y generar condiciones adecuadas para la obtención de resultados favorables" (Chiroleu, 2009: 144).

Las opiniones respecto a las fortalezas del PROGRESAR son diversas. Para muchos de las y los usuarios, el programa tiene como virtud fundamental la inclusión educativa. Como sabemos, en la Argentina, "la condición existente acerca del ingreso directo e irrestricto a las Instituciones de Educación Superior de gestión pública y la gratuidad de las mismas son consideradas por sí mismas políticas inclusivas" (Gandini, et al. 2015: 8). Sin embargo el programa, al igual que otras políticas educativas, complementa el acceso a niveles del sistema, promoviendo así la inclusión.

Una de las fortalezas tiene que ver con la política inclusiva del PROGRESAR para aquellos que, sin la prestación económica, no tenían posibilidades de acceso a estudios de nivel superior. Entonces, desde la mirada de los actores, el programa es una motivación para iniciar, 
permanecer y terminar los estudios superiores con la ayuda de la prestación económica del programa.

Otra de ellas tiene que ver con la posibilidad de insertarse en una institución de educación superior no universitaria, y permitir que el joven adquiera competencias para lo cual se forma. EI PROGRESAR sería el boleto de ingreso, permanencia y egreso a instituciones formativas habilitantes de credenciales que posibiliten perspectivas laborales a futuro. Asimismo se advierte la importancia de la obtención de un título para tener más posibilidades en el mercado laboral. El programa, entonces, tiene una impronta educativa y oportunidad laboral para los jóvenes metanenses.

Otra de las fortalezas que permite garantizar la inclusión social y educativa tiene que ver con la compatibilidad de otras políticas educativas con el PROGRESAR. Tal es el caso del boleto gratuito estudiantil interurbano (1), política jurisdiccional que se implementó en Salta en 2014, destinado a estudiantes de nivel superior universitario y no universitario, que deban trasladarse de una localidad a otra con fines académicos.

Se evidencia la finalidad de la inclusión para aquellos estudiantes que debían solventarse los gastos de transportes para realizar las Prácticas docentes y prácticas profesionales en instituciones asociadas (2). Entonces, los actores evidencian la fortaleza del PROGRESAR para cumplimentar con su formación académica de grado.

La intención de la política inclusiva del PROGRESAR apunta a que los jóvenes "recuperen espacios institucionales para expresarse dentro del ámbito de las estructuras tradicionales" (Rodríguez Use y Giambroni, 2015: 165), como ser las instituciones educativas. En un contexto globalizado, altamente hostil para las y los jóvenes, se generan profundos desafíos en políticas públicas inclusivas.

Si bien en la última década se avanzó lo suficiente para otorgar prioridad a las políticas juveniles, aún persisten desafíos por cumplir. Y esto se debe a que la categoría juvenil es abordada desde una multiplicidad de enfoques $y$, por lo tanto, no existe un modelo único de políticas juveniles que atiendan, en su integridad, todas las problemáticas.

Por último, puede evidenciarse que la mayoría de los estudiantes entrevistados han logrado acceder a la prestación económica del PROGRESAR cuando aumentó el monto de ingresos familiares como requisito para aspirar al programa. Esto significa que con el surgimiento de dicha política, en 2014, eran pocos los jóvenes quienes cumplían con los requisitos establecidos, incrementando el acceso al programa en abril de 2015.

\section{Las opiniones de los actores respecto a las debilidades del programa en relación al acompañamiento y seguimiento académico de las y los usuarios en el Instituto}


Las opiniones de las y los estudiantes usuarios respecto a las debilidades del PROGRESAR coinciden en un solo punto: la flexibilidad y la falta de control académico para mantener y renovar el derecho. Esto es, que no existe ningún mecanismo que controle o realice un seguimiento académico.

Según el Decreto 84/2014, el Comité Ejecutivo del PROGRESAR tiene el objeto de impartir instrucciones para la ejecución del mismo, así como su seguimiento y evaluación. Se desconocen los mecanismos de funcionamiento de dicho Comité para regular la política del PROGRESAR.

Dos meses después de decretado el PROGRESAR, en marzo de 2014, se llama a concurso público el cargo de Coordinador institucional de Políticas Estudiantiles (CIPEs). La Dirección de Educación Superior determina el número de un coordinador para el IES Nro. 6021. Dicho actor tiene como función aportar a la población estudiantil información con respecto a las políticas estudiantiles, como así también acompañar las trayectorias formativas estudiantiles. La Dirección de Educación Superior determina el número de un coordinador para el IES Nro. 6021.

Pese a ello, la figura del Coordinador de políticas estudiantiles no ha sido considerada como autoridad a cargo del seguimiento y acompañamiento del PROGRESAR en el Instituto de referencia. Cuando se indaga respecto al seguimiento académico, las y los jóvenes usuarios refieren a cuestiones de certificados y firmas del formulario para presentar al organismo correspondiente (ANSES). Tal como lo expresa la Vicerrectora del IES, la cuestión burocrática estaba en manos de la secretaria auxiliar y la consiguiente firma de la autoridad máxima.

Por otro lado, cuando se interroga sobre el acompañamiento o seguimiento académico, los mismos no reconocen al CIPEs como agente que cumple este rol. Asimismo, evidencian el desconocimiento de las funciones que cumple el mismo y lo relacionan con las elecciones anuales del Centro de Estudiantes.

Con respecto al seguimiento y acompañamiento del programa, concluyen que siempre se manejaron solos a la hora de informarse y gestionar el trámite para la adjudicación de la prestación económica, siguiendo el mecanismo anteriormente manifestado por la vicerrectora. Se puede inferir otra de las debilidades que tiene el PROGRESAR a nivel institucional.

Ante la falta de control y seguimiento, como mecanismo que permitiría verificar que el o la estudiante-usuario cumplimente con la finalidad educativa y formativa del programa, surgen las manifestaciones y cuestionamientos referido a la flexibilidad del programa. Asimismo, en la mayoría de los relatos, las y los usuarios comparan entre aquellos que perciben el derecho y se comprometen a asistir a clases, rendir parciales y finales, y aquellos que acuden al instituto cada vez que tienen que renovar el formulario, previo requisito para percibir el monto económico. 
Por otro lado, las y los jóvenes usuarios al referirse a la falta de control por parte del programa, comparan al mismo con otras políticas estudiantiles a las que pueden acceder. Esto es, beca estímulo, beca provincial, entre otras. Ellos distinguen los requisitos, el seguimiento y el control de dichos programas en relación con el PROGRESAR, manifestando la flexibilidad de acceso a este último. Como respuesta, sugieren la implementación de un control para aquellos usuarios que cobran el PROGRESAR y no estudian, para otorgarle transparencia al mismo. Así, se evidencia una de las debilidades más importantes del programa, que pone en cuestionamiento los límites de la aparente inclusión educativa.

\section{Un antes y un después del PROGRESAR: el estilo institucional antes del programa y después del mismo}

El acceso a la educación superior en general y de la educación superior no universitaria en particular, ha sido en nuestro país de carácter abierto y sin restricciones, fundamentando este principio en criterios de democratización de la educación. La modalidad de ingreso que adopta ha sido el de "ingreso irrestricto, con curso de apoyo y nivelación, generalmente de orientación y reflexión de tipo cognitivo; de transmisión de técnicas de estudio y de habilidades de pensamiento" (Fernández Lamarra, 2002: 63). Dicha modalidad facilita la incorporación de jóvenes provenientes de sectores vulnerables, fundamentándose en los principios de inclusión educativa.

Todo análisis del fenómeno institucional nos aventura a la complejidad de su trama. El PROGRESAR surge en 2014. Al momento de la selección de informantes claves se consideró que los mismos sean usuarios del programa, y hayan ingresado al IES Nro. 6021 antes de su implementación, para poder apreciar, desde sus relatos, la incidencia del programa a nivel institucional. En este sentido, se manifiestan dos aspectos a considerar: la matrícula estudiantil y la infraestructura edilicia.

Uno de los aspectos que sobresalen en las voces de las y los jóvenes usuarias y usuarios se relaciona con la infraestructura edilicia que albergó a 846 estudiantes durante 2015 (3). En este sentido destacan el deterioro de la institución ante la incapacidad estructural de albergar a un número de matrícula tan alto. Pese a que es un número variable, dado que al concluir el ciclo académico el número es distinto, se demuestra que en las instituciones educativas en general, y en el Instituto de Educación Superior Nro. 6021 en particular, las condiciones de trabajo no son favorables.

Esto es una cuestión que supera la finalidad del PROGRESAR, pero existen certezas de que dicha política no fue acompañada de refacción y acondicionamiento de la infraestructura. Hay que tener en cuenta que el Instituto comparte el edificio con dos instituciones educativas: una 
institución de nivel primario y otra de nivel secundario. El primero ocupa el espacio en el turno tarde y el segundo en ambos turnos, dificultando el espacio físico para el nivel superior.

Por otro lado, las y los estudiantes manifiestan que la matrícula -antes de la implementación del PROGRESAR- no se sostenía, sino a partir de 2014. Esto evidencia la incidencia del programa en la vida institucional del IES Nro. 6021. Además observan la diferencia entre otros institutos de educación superior aledaños a la localidad de Metán, afirmando que el 6021 se ha caracterizado por tener una matrícula considerable en relación a esos otros.

El Decreto 84/14 que pone en vigencia el programa PROGRESAR expresa en su artículo 15, que el Ministerio de Educación:

\footnotetext{
Deberá realizar acciones en el marco de su competencia a fin de que las distintas jurisdicciones logren, en función de sus concretas posibilidades, el objetivo de garantizar las condiciones para el ingreso, la reinserción y permanencia de los titulares de la prestación en el sistema educativo (PEN, 2014).
}

En cuanto a la infraestructura, cabe destacar que los actores no evidencian cambios favorables, dado que en el predio en donde funciona el IES Nro. 6021 no hubo modificaciones después de la implementación del PROGRESAR. Mantienen un discurso negativo respecto de las instalaciones del instituto, destacando el aumento de la matrícula y las condiciones estructurales en las que cursan sus estudios. Estos aspectos (matrícula y condiciones edilicias) han incidido en el estilo institucional en cuanto al cursado de las materias, la disponibilidad de mobiliario, las condiciones higiénicas, la saturación de las aulas, lo que causa un malestar en los estudiantes.

En relación con ello y siguiendo con los lineamientos del decreto del PROGRESAR, debemos considerar que el Ministerio de Educación deberá dar respuesta al impacto del potencial aumento de la matrícula en el ya colapsado sistema de educación pública, sobre todo, en lo relativo a la reinserción de jóvenes adultos. En este sentido:

(...) es posible que el no cumplimiento de las condicionalidades del programa supere la voluntad de los destinatarios, y más bien se asocie a problemas generalizados de recursos y capacidades del sistema educativo, como falta de vacantes y de infraestructura para absorber a la nueva demanda (Marzonetto y Aguirre, 2014: 20).

En síntesis, el decreto nacional que autoriza la creación del PROGRESAR posee grandes falencias respecto a otro tipo de políticas que acompañan al programa. Puede evidenciarse que no existen documentaciones que establezcan criterios referidos a las instrucciones para la puesta en marcha del programa, ni los recursos necesarios para sostener dicha política en los institutos de educación superior no universitaria. Se puede afirmar que el PROGRESAR no fue 
acompañado por otras medidas que permiten una política efectiva del mismo. Sumado a ello, se obstaculiza con las y los jóvenes usuarios que cobran el programa y sus trayectorias formativas son discontinuas (Terigi, 2010). Esto permite inferir que la inclusión es relativa.

\section{La incidencia del programa en la trayectoria académica de las usuarias y los usuarios y las sugerencias/ modificaciones respecto del PROGRESAR}

Pensar las trayectorias como un "itinerario en situación" (Nicastro y Greco, 2012: 25) de cada uno de los sujetos, nos permite visualizar respecto a cómo cada uno utiliza -por no decir, aprovecha- el monto económico del programa para fines educativos. Si bien el PROGRESAR tiene como objetivo brindar oportunidades educativas y laborales a los jóvenes, el mismo no manifiesta los mecanismos de control para que sea efectivo, y mucho menos indica cuáles son las condiciones académicas necesarias para promover oportunidades educativas inclusivas.

Se evidencia que la mayoría de los usuarios del programa no lo utilizan con la finalidad educativa con la que surge. Esto se debe a que el programa no exige ningún tipo de rendimiento académico (pasar de año, mantener un promedio, aprobar una determinada cantidad de materias al año, etcétera), sino que permite permanecer en el sistema educativo. $Y$ es allí donde las y los usuarias y usuarios sugieren modificaciones, como por ejemplo, el control académico para poder cobrar el derecho, permitiendo que el Estado ahorre esa plata en jóvenes que no estudian y que asistan al instituto los que tienen intenciones de formarse y superarse académica y profesionalmente.

Los cambios y modificaciones que se deben considerar, desde las voces de los actores, giran en torno al control. La normativa que pone en marcha al PROGRESAR no específica cómo los diversos organismos que articulan dicha política, garantizan el acceso real y flexible a la oferta educativa. Tampoco aclara cómo se llevará a cabo esta intervención, lo cual permite que el derecho resulte laxo para las jóvenes y los jóvenes que cumplen con las mínimas condiciones requeridas, estudien o no.

Entonces, modificando los mecanismos de acceso y control del programa condicionaría al estudiante a transitar un recorrido académico formativo a conciencia, utilizando el programa para fines educativos. Los encargados de la modificatoria serían los CIPEs que, trabajando de manera conjunta con el cuerpo de gestión, realizarían un seguimiento de las trayectorias académicas formativas, un acompañamiento respecto al rendimiento y la promoción de una política juvenil que esté destinada, en cuanto a inclusión educativa, a que el usuario con el PROGRESAR obtenga un título de Educación Superior no Universitario.

\section{Resultados}


Los actores institucionales manifiestan que el PROGRESAR posee relevancia para aquellos jóvenes que no disponían de recursos económicos para solventar los estudios de nivel superior. El acceso a una carrera en un instituto de educación superior no universitario se le atribuye, en gran medida, al programa. Asimismo, debido a la oferta educativa de las instituciones de nivel superior en la localidad de Metán y la posibilidad de acceso de los jóvenes al PROGRESAR, permitió que los mismos inicien y continúen una carrera superior con la intención de obtener un título que les permita acceder al mercado de trabajo.

Por otro lado, el programa tuvo incidencia en la vida familiar de los usuarios dado que en la mayoría de los casos se demostró que la familia realiza un acompañamiento y estimula a los jóvenes estudiantes a permanecer en el sistema y recibirse. En base a ello se evidencia la mirada que tiene la familia de los usuarios frente al programa, ya que, desde su opinión, les permitió alivianar los gastos destinados a los estudiantes que se forman en una institución.

Cuando nos referimos a la inclusión como finalidad del programa, los actores advierten que hubo inclusión, ya que muchos accedieron a estudios superiores. Pero esa inclusión es "relativa" dado que no todos los usuarios utilizan el monto económico del programa con fines formativos. En este caso, la mayoría evidencia la flexibilidad del programa en cuanto a los requisitos básicos que solicita la política juvenil para acceder al derecho, obstaculizando una inclusión educativa efectiva.

Estos actores sugieren, como modificación que permitiría garantizar la inclusión educativa y social, implementar mecanismos de control para el acceso al programa. Tales mecanismos serían: cantidad de materias aprobadas/finalizadas por año; seguimiento regular de los usuarios; promedio general mínimo; asistencia a clases, entre otros.

En este sentido, como política juvenil que apunta a facilitar el ingreso, la permanencia y el egreso y obtención de un título de educación superior, se equipararía a otros estímulos económicos que promueven una trayectoria académico-formativa exitosa. Estos mecanismos de control, que contribuirían al seguimiento y rendimiento académico de los usuarios del derecho, acompañado de actores institucionales que se comprometan en la implementación real de una política juvenil y nacional, permitirían un lineamiento nacional transparente y eficaz que contribuya a la inclusión social y educativa que propone el PROGRESAR.

En relación con la incidencia del PROGRESAR en la vida institucional, puede señalarse que la infraestructura edilicia no está preparada para albergar a una población estudiantil numerosa. Pese a ello, las autoridades debieron realizar gestiones para poder cubrir la demanda con los recursos limitados.

En el edificio del Instituto de Educación Superior Nro. 6021 funcionan dos niveles educativos más. Esta idiosincrasia es compleja, dada la interposición de horarios entre las horas de clases del nivel secundario y el nivel superior. La creación de comisiones en el IES se ve limitada por 
la falta de espacio edilicio. Esto genera un malestar recurrente entre los estudiantes, las autoridades y la falta de respuesta ante la necesidad de un edificio propio.

La incidencia del PROGRESAR ha modificado significativamente el estilo institucional, especialmente en el caso del IES Nro. 6021. Los aspectos estructurantes del mismo se han complejizado, debido al aumento de matrícula que se evidenció desde la implementación del PROGRESAR. Por otro lado, y en relación con ello, los actores manifiestan la urgencia de atender a las necesidades primordiales que padecen institucionalmente. Asimismo, se demuestra que el programa no estuvo acompañado de políticas que apunten a remodelar y ampliar los edificios donde funcionan los institutos de educación superior.

Entonces, en paralelo a la implementación de políticas educativas que permiten el acceso a sectores no pudientes a la educación superior, y el impacto que genera el aumento significativo de matrícula estudiantil, se deben atender las necesidades y demandas edilicias para garantizar la política inclusiva en educación superior. El control y el seguimiento por parte de la Dirección de Educación Superior en acuerdo con el Ministerio de Educación, Ciencia y Tecnología, son obligaciones prioritarias para cumplimentar con las líneas políticas vigentes.

Puede evidenciarse la incidencia del PROGRESAR a nivel institucional, a nivel social y a nivel familiar y académico. Los actores destacan la impronta del programa, las debilidades y las sugerencias que desde su perspectiva realizarían. Los jóvenes son conscientes de la incidencia del programa y los efectos que resultan del mismo. Una política inclusiva desde la mirada juvenil debe estar acompañada de un seguimiento a nivel macro y micro, y considerarse prioritaria en la agenda educativa nacional.

Dado que en la presente investigación se trabajó desde un estudio de casos, esto permite interrogarnos sobre la incidencia del PROGRESAR en otras instituciones de educación superior de la provincia, en particular, y del país en general. La mejora consiste en revisar las políticas educativas que apuntan a las problemáticas juveniles, mediante un trabajo articulado de los ministerios implicados en el programa.

Por otro lado, si bien la educación superior en la Argentina se caracteriza por ser de ingreso irrestricto y gratuita, esto no implica que el joven que desee ingresar a los niveles de educación superior no invierta económicamente durante todo su trayecto formativo profesional. Si bien las condiciones socioeconómicas de los jóvenes de 18 a 24 años les permiten acceder al PROGRESAR, no es este requisito suficiente para garantizar el ingreso, la permanencia y el egreso de esta franja etaria al sistema de educación superior. Las condiciones académicas de los jóvenes también determinan las trayectorias transicionales de los mismos (Terigi, 2010). Este requisito es fundamental para poder garantizar la permanencia en el nivel y el posterior egreso del mismo.

El análisis realizado en la presente investigación se basa en un estudio de casos. La misma muestra que la incidencia del PROGRESAR es una cuestión compleja dado que intervienen 
múltiples dimensiones, evidenciando las potencialidades del mismo como así también sus limitaciones. Se espera que el presente trabajo aporte al estado de la cuestión para futuras investigaciones.

\section{Notas}

(1) El pase libre es una política provincial y se caracteriza por la entrega de boletos gratuitos a estudiantes del nivel que acrediten certificado de alumno regular.

(2) Se denominan instituciones asociadas a un conjunto de instituciones educativas y laborales que permiten el acceso a estudiantes para realizar las prácticas profesionales.

(3) Según datos ofrecidos por la autoridad institucional, se evidencia que en 2013 la matrícula fue de 657; en 2014 fue de 792 y en 2015, 846 inscriptos.

\section{Bibliografía}

Chiroleu, A. (nov. 2011). La Educación Superior en América Latina: ¿problemas insolubles o recetas inadecuadas? Avaliação, Campinas; Sorocaba, SP, v. 16, n. 3, pp. 631-653.

Cols, E. (2008). La formación docente inicial como trayectoria (mimeo). Documento de Trabajo, Ciclo de Desarrollo Profesional de Directores.

Dávila León, O. (diciembre 2004). Adolescencias y juventud: de las nociones a los abordajes. Revista Última década, 21, CIDPA, Valparaíso, pp. 83-104.

Poder Ejecutivo Nacional (PEN) (2014). Decreto 84/2014. Programa de Respaldo a Estudiantes Argentinos (PROGRESAR). Boletín Oficial de la República Argentina, CXXII(32.814).

Fernández Lamarra, N. (2002). La educación Superior en Argentina. IESALC/UNESCO Instituto Internacional para la Educación Superior en América Latina y el Caribe Ministerio de Educación, Ciencia y Tecnología de la República Argentina, Secretaría de Políticas Universitarias.

Recuperado

de http://unesdoc.unesco.org/images/0014/001494/149464so.pdf

Gandini, M.; Maldonado, L. y Moreno Yunis, D. (2015). PROGRESAR: ¿políticas de ayuda a la inserción de los jóvenes en el sistema de educación superior argentino? Recuperado de https://www.researchgate.net/publication/270890722

Gutiérrez, A. (2002). Pierre Bourdieu. Las prácticas sociales. Buenos Aires: Centro Editor de América latina.

Margulis, M. y Urresti, M. (1996). La juventud es más que una palabra. Ensayos sobre cultura y juventud. Buenos Aires: Editorial Biblos. 
Marzonetto, G. y Aguirre, J. (2014). Sin estudio y sin trabajo. El Programa de Respaldo a Estudiantes Argentinos: PROG.R.ES.AR. Centro Interdisciplinario para el Estudio de Políticas Públicas, Serie Análisis de Coyuntura, 25.

Nicastro, S. y Greco, M. (2012). Entre trayectorias. Escenas y pensamientos en espacios de formación. Rosario: Homo Sapiens Editorial.

Rodríguez Gómez, G.; Gil Flores, J. y García Jiménez, E. (1996). Metodología de la investigación cualitativa. Málaga: Aljibe.

Rodríguez Use, J. y Giambroni, L. (2015). Avances y desafíos de las políticas de juventud del siglo XXI. Revista Estado y Políticas Públicas, 4, pp. 152-165.

Terigi, F. (23 de febrero de 2010). Las cronologías de aprendizaje: un concepto para pensar las historias escolares. Jornada de Apertura del ciclo lectivo 2010, Santa Rosa. Recuperado

de

http://www.chubut.edu.ar/concurso/material/concursos/Terigi_Conferencia.pdf 